\title{
Kompressionsstrümpfe lindern schnell Schmerzen
}

Kakkos S et al. Acute Effects of Graduated Elastic Compression Stockings in Patients with Symptomatic Varicose Veins: A Randomised Double Blind Placebo Controlled Trial. Eur J Endovasc Surg 2018; 55: 118-125. doi:10.1016/ejvs.2017.10.004

Der Nutzen von graduierten Kompressionsstrümpfen (GECS) bei Patienten mit primären Varizen ist umstritten. Die griechische Arbeitsgruppe überprüfte daher nun den kurzfristigen Nutzen bei Erkrankten, die noch keine GECS benutzt hatten und keine oberflächliche Thrombose, Ulcera sowie Juckreiz aufwiesen. In der Doppelblindstudie erhielten je 15 Patienten GECS Klasse 1 (1821 mm HG auf Knöchelniveau) oder Placebo gleichen Aussehens. Wenn beide Unterschenkel betroffen waren, war der schwerer erkrankte das Indexbein. Primärer Endpunkt war die Schmerzreduktion nach 1 Woche, die auf einer visuellen Analogskala erfasst wurden. Weitere Endpunkte waren u.a. Schweregefühl, Schwellung, Brennen, Parästhesien, Knöchelumfang und der Wert auf der modifizierten VCSS-Skala (Venous Clinical Severity Score). Referenzstandard war das kontralaterale Bein.
Pro Gruppe brach 1 Patient die Teilnahme ab. Die Basisdaten der Gruppen waren vergleichbar. Meistens war die V. saphena magna betroffen und überwiegend bestand eine CEAP-Klasse 2. Verglichen mit Placebo reduzierten die GECS signifikant effektiver die Schmerzen. In 9 Fällen trat eine vollständige Rückbildung und bei 3 Erkrankten eine Abnahme der Beschwerden ein. 11 Patienten wiesen eine Schmerzreduktion $>50 \%$ auf. Mit Placebo hatten 2 Patienten keine Schmerzen mehr und 5 empfanden eine Linderung. Tendenziell beeinflussten GECS auch andere Beschwerden. Der initiale und 7-Tage-Knöchelumfang unterschieden sich am Index- und kontralateralen Bein nicht. Jeweils 1 Patient berichtete über Juckreiz und neu aufgetretene Oberschenkelkrämpfe, wobei sich gleichzeitig Unterschenkelkrämpfe vollständig zurückgebildet hatten. Die Compliance war ins- gesamt hoch. Die tägliche Nutzungsdauer betrug in der aktiven Gruppe 10,2 Stunden und in der Placebogruppe 8 Stunden. Zwischen der Tragezeit und der absoluten Schmerzreduktion auf der VAS-Skala bestand keine Assoziation.

Die Studie belegte somit den kurzfristigen Nutzen der graduierten Kompressionsstrümpfe, erlaube aber keine Rückschlüsse auf die langfristige Effektivität, so die Autoren. Die Compliance dürfte über längere Zeiträume eher abnehmen, v.a. wenn Beschwerden rückläufig seien. Auch die geringe Patientenanzahl insgesamt und mit höheren CEAP-Klassen schränke die Aussagekraft der Ergebnisse ein. In jedem Fall halten Kakkos et al. Langzeitstudien für gerechtfertigt.

Dr. med. Susanne Krome, Melle 